\title{
Enquêter sur les formations d'ingénieurs en France : la construction d'une catégorisation sur la place et la fonction des sciences humaines et sociales
}

\author{
Catherine Roby ${ }^{1}$ et Brigitte Albero ${ }^{2}$ \\ ${ }^{1}$ Doctorante sous contrat doctoral à l'ED SHS 507 (CREAD, EA 3875) \\ ${ }^{2}$ Professeur des universités, co-directrice du CREAD (EA 3875)
}

\begin{abstract}
Résumé. Les formations d'ingénieurs en France sont reconnues comme relevant d'une grande diversité. Est-il possible cependant de les comparer sur le terrain de leurs pratiques de formation? Quels critères privilégier et comment s'y prendre en toute rigueur ? Quels sont les enjeux d'une telle enquête ? Cette contribution aborde ces questions en présentant la première partie d'une enquête qui, à partir de l'étude des programmes de formation mis en ligne sur les sites internet de l'ensemble des Ecoles en France, débouche sur la construction d'une catégorisation des formations centrée sur la place et la fonction des SHS dans ces formations. Elle conclut sur les perspectives de recherches empiriques ainsi ouvertes.
\end{abstract}

\begin{abstract}
It is recognised that engineering training courses in France vary enormously. Is it nevertheless possible to make comparisons between them? And if so, using what criteria, why and how? What are the challenges? This article attempts to answer these questions by outlining the implementation and results of the first part of a survey conducted on the training courses put online on the internet sites of the all French engineering schools. This survey led to a categorization of training courses based on the place and function of humanities and social sciences (HSS) in schools. This article as well suggests new directions for empirical research into the problematic issue of HSS in engineering training.
\end{abstract}

Les Écoles d'ingénieurs constituent en France un paysage contrasté au point que l'on pourrait juger vaine leur comparaison. La genèse spécifique de ce champ de pratiques, la socio-histoire de chaque École liée à la socio-histoire de chaque branche professionnelle et de leurs relations respectives sur un territoire particulier est si singulière que l'enquêteur est tenté d'en dresser autant de monographies. Pourtant l'enquête nationale sur l'ensemble des Écoles trouve son sens dans la centration sur un dénominateur commun exploité en tant qu'analyseur: la place et la fonction que les pratiques de formation des Écoles attribuent aux sciences humaines et sociales (SHS). Cette enquête porte sur les affichages des disciplines de SHS proposés dans les cursus de formation présentés sur les sites internet des Écoles, ainsi que sur l'analyse des discours qui accompagnent ces présentations.

Après avoir fourni quelques éléments de contexte visant à situer le paysage national des Écoles d'ingénieurs en France et les enjeux d'une comparaison, cette contribution présente la méthodologie

This is an Open Access article distributed under the terms of the Creative Commons Attribution License 4.0, which permits unrestricted use, distribution, and reproduction in any medium, provided the original work is properly cited. 
d'élaboration d'une catégorisation conçue non comme un outil d'évaluation des pratiques de formation mais comme un instrument permettant de comprendre les dynamiques en jeux et les logiques à l'œuvre. Cette visée d'intelligibilité devrait permettre d'actualiser le débat sur le rapport entre les connaissances produites par les SHS et la conduite de formations aux fonctions d'encadrement et de décision, quelles que soient les spécialités. Plus largement, c'est aussi le débat sur les relations entre culture technique et humanités qui peut ainsi être documenté, dans une période où les savoirs techniques passent encore pour ancillaires et neutres.

\section{La place et la fonction des SHS dans les formations des ingénieurs en tant qu'analyseur : les enjeux d'une comparaison}

La prise en compte de la genèse particulière des formations d'ingénieurs en tant que champ de pratiques, le constat de la diversité justifiée des situations locales, l'analyse des relations complexes entre disciplines constituent à elles seules des pistes d'enquête pour autant d'études historiques, sociologiques, épistémologiques. Sans négliger ni leur intérêt ni leur importance, il apparaît néanmoins que l'étude des intitulés des enseignements tels qu'ils sont présentés par les Écoles dans leurs programmes de formation constitue un analyseur pertinent pour un premier repérage concernant la place et la fonction accordées aux SHS dans ces programmes.

\section{Genèse d'un champ de pratiques et constat de sa diversité}

Dans la formation des médecins, l'apparition d'enseignements de SHS est récente. En 1992, un arrêté ministériel a rendu la culture générale obligatoire pendant la première année des études médicales, conduisant à l'introduction de cours d'expression-communication, de philosophie et d'histoire des sciences. Ces enseignements étaient alors pris en compte pour au moins $10 \%$ dans la sélection à l'entrée en seconde année. Ce coefficient est passé à $20 \%$ en 1995, pour des enseignements intitulés « sciences humaines et sociales. » Depuis lors, les dénominations de ces enseignements se sont diversifiées en « santé », « société », « humanités » et les enseignants de SHS en médecine et santé disposent d'un collège pour se concerter (Visier, 2011).

Dans la formation des ingénieurs, il en va tout autrement. Les humanités ont très tôt fait partie des enseignements, au cours du premier développement des Écoles au XIX ${ }^{\text {ème }}$ siècle. La culture générale étant alors considérée indispensable aux ambitions intellectuelles, professionnelles et sociales de ces ingénieurs généralistes appelés aussi par leurs fonctions à préserver les pouvoirs politiques en place. Au début du XXème siècle, l'apparition des ingénieurs spécialisés destinés à accompagner les développements industriels dans ses différentes branches relève d'un tout autre profil avec une formation exclusivement scientifique et technique qui ne laisse place en matière d'intérêt pour les humanités qu'à quelques enseignements relatifs à l'hygiène et à la sécurité au travail. Environ deux cents nouvelles Écoles sont créées au cours du $\mathrm{XX}^{\text {ème }}$ siècle à partir de ces premiers modèles.

Aujourd'hui, l'ensemble de ces Écoles, plus généralistes ou plus spécialisées, coexistent dans un paysage qui s'est beaucoup diversifié. Elles se distinguent en particulier par leurs domaines d'intérêt ${ }^{1}$,

\footnotetext{
${ }^{1}$ Sciences des systèmes industriels, des procédés chimiques/biochimiques, du traitement du signal et de l'information, de la matière, du vivant et de l'environnement.
} 
leur mode de recrutement ${ }^{2}$, mais aussi par leurs tutelles ${ }^{3}$. Face à cette diversité se pose alors la question de la connaissance que l'on peut en produire. Mais se pose aussi la question des pratiques effectives de formation et notamment celle des liens qui existent ou non entre les sciences de la nature et des techniques d'une part et les sciences de l'humain et du social d'autre part.

\section{Écoles d'ingénieurs et SHS : une relation qui peine à s'instituer}

Si les SHS produisent peu de travaux sur les Écoles d'ingénieurs malgré les enjeux d'une telle connaissance, celles-ci quant à elles tendent globalement à négliger l'importance des savoirs produits par les SHS dans la formation de professionnels à fonction d'encadrement et de décision.

En ce tout début du XXI ${ }^{\text {ème }}$ siècle, la question des «Humanités », des «SHS pour l'ingénieur » ou ce qui est encore appelé la « Formation Humaine » a fait l'objet en Sciences de l'éducation de seulement deux thèses, de ce fait pionnières ${ }^{4}$ (Dufour, 1998 ; Lemaître, 2001). Ces travaux conduits par des enseignants en poste dans des Écoles, posent les bases d'une réflexion sur les incidences de tels enseignements. D'autres travaux en histoire des sciences, sociologie ou encore sciences politiques apportent des éléments de compréhension sur la place de ces formations dans les Écoles et leurs relations avec les autres diciplines. Malgré l'intérêt des études existantes, leur rareté et leur dispersion ne peuvent qu'être soulignées (Rollet, 2009) dans une période où les débats sur la formation des ingénieurs retrouvent une certaine vigueur (AERES, 2010 ; Bordier, Kirchner, Nussbaumer, 2011 ; ISAE Executive Club, 2011 ; Sainseaulieu, Jammet, 2011) et où la complexification des situations de travail qui relie le champ des sciences et des techniques à ceux de l'économique et du politique recouvre de forts enjeux.

De manière symétrique, les SHS développent de longue date des connaissances qui documentent le rapport que l'humain entretient à la science et à la technique (Albero, 2004, 2010), sans que pour autant de telles connaissances soient enseignées. Depuis la décennie 1970, l'histoire et la sociologie des sciences et plus récemment, les disciplines qui analysent l'activité humaine (éducation \& formation, gestion \& management, information \& communication, santé, travail, sports) produisent des connaissances qui tendent à relier rationalité des sciences et des techniques et perspectives épistémiques d'une autre nature (culturelle, politique, économique, éthique). Par ailleurs, les connaissances relatives aux organisations et au travail, produites en sociologie, psychosociologie, économie, gestion devraient s'avérer utiles dans un cursus d'ingénieur.

La question qui se pose est donc de savoir quelle place les cursus accordent aux connaissances issues des recherches en SHS, comment les différentes formations qualifient de tels enseignements et selon quels principes de pertinence structurer une catégorisation qui ait du sens pour la recherche en SHS, tout en constituant un instrument recevable par les Écoles en vue de son utilité dans l'analyse et l'évolution des curricula.

\section{Les conditions de l'enquête sur les modalités d'affichage des SHS dans les formations d'ingénieurs : cadre théorique et méthode}

L'étude des programmes de formation tels qu'ils se donnent à lire sur les supports de communication que sont les sites internet et les plaquettes sur support papier constitue une première approche de

\footnotetext{
2 De bac à bac+4, concours après deux années de classes préparatoires, par concours ou sur dossier et entretiens après le baccalauréat ou encore, mais plus marginalement, sur titre, pour des titulaires de brevets de technicien supérieur, diplôme universitaire de technologie, licences ou masters.

${ }^{3}$ Ministères techniques, enseignement supérieur et recherche, avec des Écoles internes aux universités ou indépendantes, Écoles consulaires, privées, confessionnelles.

${ }^{4}$ Ces thèses sont présentées et rapprochées dans un article soumis à la revue TransFormations: Roby C., Albero B., Formation des ingénieurs et SHS : les apports de deux thèses pionnières en sciences de l'éducation.
} 
l'ensemble des Écoles du territoire national ${ }^{5}$. L'analyse de ces programmes trouve ses fondements dans la sociologie du curriculum et elle a été conduite par approches successives selon une méthode d'abord exploratoire puis de plus en plus systématique jusqu'à saturation de l'information à partir d'une succession d'hypothèses à fonction heuristique.

\section{La sociologie du curriculum en tant que ressource conceptuelle}

Développée en Grande Bretagne par B. Bernstein et M. Young au début des années 1970, la sociologie du curriculum a été diffusée en France dans les décennies 1980-1990. En sciences de l'éducation, les travaux de V. Isambert-Jamati, P. Perrenoud et J-C. Forquin ont largement contribué à cette diffusion.

Dans son sens le plus général, le terme «curriculum » fait référence aux processus conjoints, à la fois cognitifs et culturels de l'acte d'enseignement, en englobant dans la transmission de savoirs et d'aide au développement de connaissances et de compétences, les représentations et les valeurs qui lui sont également attachées (Forquin, 2008). En étudiant les formes concrètes de cette manifestation, ce courant de recherche délimite le curriculum aux programmes d'étude tels qu'ils apparaissent par les appellations et les modalités d'organisations des contenus d'enseignements. Les travaux mettent en valeur le caractère socialement construit du curriculum dans un processus d'élaboration permanent qui mobilise des ressources humaines et matérielles dans des choix et orientations. L'étude de leurs matérialisations permettent de repérer comment se distribuent les systèmes d'intérêts et de valeurs et donc les rapports de force. Le curriculum constitue ainsi un analyseur pertinent des choix qui finissent par s'instituer, définissant des territoires et des frontières, conférant des cultures et des identités. C'est ainsi que la sociologie du curriculum étudie la hiérarchisation des savoirs et des disciplines, les marques de leur prestige, de leur reconnaissance sociale et leur poids dans les évaluations.

Les principaux apports de ce courant de recherche relèvent de la différenciation de trois phénomènes (Forquin, 2008) : 1) le « curriculum formel » correspond aux prescriptions des programmes officiels et apparaît dans les maquettes de formation ; 2) le « curriculum réel » correspond à la pratique effective des enseignants et apparaît dans les modalités pédagogiques explicites ; 3) le «curriculum caché » correspond à ce qui est transmis par imprégnation, familiarisation, inculcation diffuse, socialisation dans un certain environnement. Il a également mis en valeur la «classification » plus ou moins implicite des savoirs et donc des disciplines par l'organisation même de leurs modes de transmission, faisant apparaitre deux « codes » distincts : a) le « code sériel » qui désigne une forte compartimentation des savoirs disciplinaires organisant ainsi une juxtaposition des enseignements et des contenus de la formation ; b) le code «intégré » qui désigne des formes pédagogiques mobilisant plusieurs disciplines dans l'étude d'un même objet. Ces deux codes qui sous-tendent l'organisation des curricula, induisent des variations importantes dans les identités professionnelles des intervenants, leurs relations et les formes et modalités de leurs pratiques pédagogiques.

Dans la mesure où les Écoles d'ingénieurs doivent définir, à partir des indications de la Commission des titres d'ingénieurs ${ }^{6}$ (CTI), de leur tutelle et de leurs différents partenaires, « les problèmes de définition des objectifs de formation, de constitution des disciplines et de mise en scène pédagogique, qui définissent l'organisation du curriculum selon Jean-Claude Forquin (1996) » (Sonntag et al., 2008), il apparaît donc pertinent de chercher à savoir par l'analyse des curricula formels des Écoles d'ingénieurs, quels sont les tris réalisés, les choix effectuées et, in fine, les décisions prises.

\footnotetext{
5 Écoles des Formations initiales sous statut étudiant.

6 Organisme paritaire chargé depuis 1934 de l'habilitation des formations d'ingénieurs.
} 


\section{L'élaboration d'une méthodologie d'enquête entre exploration et systématicité}

Le défi d'une étude nationale sur un tel terrain consiste à trouver l'échelle et la méthode pertinente qui permet au chercheur d'être exhaustif en restant intelligible, rigoureux en restant inventif, cohérent dans ses constructions en restant ouvert aux exceptions qui viennent régulièrement le contredire.

En première approche, l'échelle de l'étude a été délimitée par les programmes de formation tels qu'ils sont communiqués sur les sites internet des Écoles, en centrant l'analyse sur les appellations et le volume des enseignements des SHS dans la formation. La généralisation des sites internet des Écoles en vue d'assurer leur communication et d'attirer des candidats a permis d'atteindre un degré d'exhaustivité et de précision satisfaisant. Une liste de 191 établissements de formation initiale sous statut d'étudiant a été élaborée à partir du croisement de différentes sources fiables ${ }^{7}$. Elle regroupe la totalité des Écoles de ce type qui représentent 93,37 \% des établissements habilités par la CTI à délivrer un diplôme d'ingénieur. Les autres établissements délivrent des diplômes uniquement par la voie de la formation continue ou par celle de la formation initiale sous statut d'apprenti, ou encore sont des Écoles de spécialisation ${ }^{8}$. Par ailleurs, la délimitation des disciplines qui composent la référence aux « SHS » a été élaborée à partir des appellations des sections $\mathrm{CNU}^{9}$, même si, dans le contexte du terrain d'étude, il est indispensable, pour observer les dispositifs effectifs d'étendre la définition des SHS aux savoirs académiques qui concernent l'humain et ses organisations sociales, mais aussi ceux qui proviennent de la philosophie et des études littéraires et artistiques. Les langues étrangères étant toujours présentes dans les programmes de formation, elles n'ont pas été retenues en tant qu'indicateur discriminant. A ces conditions, toutes les appellations des enseignements susceptibles de faire appel, d'une manière ou d'une autre, à ces connaissances que nous qualifions dans cette contribution de «SHS » ont pu être prises en compte. Ces appellations ${ }^{10}$ ont été scrupuleusement conservées sans modification, telles qu'elles apparaissent dans les programmes de formation des Écoles.

Progressivement, quatre aspects se sont avérés constituer des indicateurs importants pour l'analyse: 1) la dénomination de ces enseignements ; 2) l'existence ou non d'une entité instituée (Département, Pôle, ou toute autre dénomination) regroupant les enseignants et éventuellement les enseignements de ces disciplines ; 3) l'inscription ou non des enseignants dans des unités de recherche et l'inscription de ces unités dans les Écoles ou à l'extérieur (ces items pouvant se combiner entre eux avec la participation d'enseignants en tant que chercheurs de SHS impliqués dans des unités de sciences de l'ingénieur de l'École) ; 4) certaines caractéristiques des Écoles (date de création, tutelle, domaine de spécialité dominant, niveau de recrutement, localisation géographique, genre des directions).

Des tris à plat et croisés ont été réalisés dans un tableur à partir des données collectées sur ces différents aspects, permettant une analyse descriptive du corpus. Ces résultats ont été corroborés par des analyses multivariées (ACP) (Roby, 2011). Ils mettent en valeur la forte relation entre les intitulés figurant dans les programmes de formation affichés sur les sites internet se rapprochant des SHS au sens le plus large et des thématiques liées au monde de l'entreprise visant des applications utilitaires. Ils sont souvent proposés sous l'appellation générique de « connaissance de l'entreprise » ou encore de «sciences de l'entreprise » et recouvrent une grande diversité d'approches. La gestion de projet,

\footnotetext{
${ }^{7}$ Liste des écoles habilitées parue au JO de 2010 ; sites internet de la CTI, de la conférence des grandes écoles, du centre d'étude des formations d'ingénieurs, guide « Hobson » des grandes écoles, numéro hors-série «Le Monde de l'éducation » de novembre 2010 portant sur les écoles d'ingénieurs.

${ }^{8}$ L'IFP School (institut français du pétrole), l'institut national des sciences et techniques du nucléaire, etc.

${ }^{9}$ http://www.cpcnu.fr/sectionsCnu.htm

${ }^{10}$ Dans l'ordre alphabétique : activités sportives ; anthropologie/ethnologie ; communication/expression ; culture générale/activités d'ouverture culturelle ; culture internationale ; développement personnel/connaissance de soi ; droit ; économie/gestion/sciences de gestion (marketing, comptabilité, etc.) ; entreprise ; éthique ; géographie ; histoire ; management des entreprises/gestion des ressources humaines ; gestion de projet/management des projets ; ouverture sociale ; philosophie ; philosophie des sciences/ épistémologie/Sciences Techniques Société ; projet personnel et professionnel ; psycho-sociologie ; psychologie/psychanalyse; sciences politiques/géopolitique ; sociologie.
} 
le management, parfois la sociologie des organisations, mais aussi les techniques d'expression et de communication, d'animation de réunion y occupent une place récurrente. Ces résultats sont cohérents avec ceux obtenus à l'échelle européenne, dans le cadre du projet HELENA (Higher education leading to Engineering and scientific carrers) (Godfroy, 2013).

Après cette étude quantitative des données, une vingtaine d'Écoles ont été choisies sur les critères d'âge, de tutelle, d'orientation de spécialité dominante, de niveau de recrutement, avérés par l'étude quantitative, en tant que facteurs de positionnement différentiel des SHS dans les Écoles. Les textes de présentation des Écoles et des formations en SHS (et assimilées) des Écoles ont été analysés par l'élaboration de schémas actanciels ${ }^{11}$. Ce travail a permis d'identifier les dominantes de ces discours de présentation et de proposer une catégorisation des Écoles sur les critères de la place et de la fonction des SHS. Ensuite, une relecture complète de tous les textes de présentation des formations de SHS et assimilées des 191 Écoles a été effectuée jusqu'à saturation des données (Strauss, 1992, 2004).

Une hypothèse initiale a permis d'organiser les résultats entre deux pôles contrastés : l'un relevant d'une absence quasi totale d'enseignements des SHS ; l'autre relevant d'une intégration en secteurs interdisciplinaires entre sciences des ingénieurs et SHS.

L'analyse structurale a permis de construire deux groupements intermédiaires : l'un ne conservant des SHS que leurs apports les plus instrumentaux (techniques de communication ou d'animation de groupe par exemple) ; l'autre en y sélectionnant des enseignements adaptés au domaine de formation (sociologie du monde rural ou sociologie du travail par exemple). Six grandes catégories se sont ainsi progressivement stabilisées, par approximations successives.

\section{Catégorisation des formations d'ingénieurs selon la place et la fonction des SHS}

L'hypothèse initiale ayant résisté à l'analyse structurale du corpus, la catégorisation présente donc un continuum en tension entre deux pôles contrastés, depuis une quasi absence d'apports explicites jusqu'à une intégration interdisciplinaire entre sciences et techniques de spécialité des ingénieurs et SHS. Les appellations sont sans doute amenées à évoluer vers une plus grande homogénéité.

La catégorie (1) intitulée «Dynamiques professionnelles » rend compte des programmes qui exploitent sans les nommer des apports génériques des SHS, en vue de développer une culture générale et un ensemble de qualités professionnelles, sans pour autant distinguer une ou des disciplines de SHS en particulier. Les interventions dans la formation sont majoritairement prises en charge par des professionnels d'entreprises partenaires, sous forme de conférences et de témoignages qui apportent un éclairage venant du monde du travail dans les domaines d'activité auxquels se destinent les élèves. Ces enseignements s'organisent souvent autour d'un module de type «projet personnel et professionnel » ou « connaissance de l'entreprise et de ses métiers ».

La catégorie (2) appelée «Culture métier » rend compte des programmes qui visent à former un ingénieur-manager susceptible d'exercer son activité dans l'environnement économique et social des entreprises. Elle regroupe des formations qui se veulent pratiques et opérationnelles, juxtaposées aux formations en sciences et techniques relevant de la spécialité visée et n'ayant aucune relation avec elles.

\footnotetext{
11 En référence à A.J. Greimas (1966a/b) repris par M. Linard (1989, 1994) dans la perspective d'une théorisation de l'apprentissage en tant qu'activité humaine instrumentée, l'analyse consiste à identifier les « actants » d'un récit (destinateurobjet-destinataire dans le registre de la « communication », éventuellement les formes de relations entre objet et sujet dans le registre du « désir » ou du « vouloir » et celles entre adjuvant et opposant dans le registre du « pouvoir »). Dans l'analyse conduite, le programme de formation qui s'affiche sur Internet est considéré comme un récit. Cela permet de repérer les caractéristiques attribuées : au destinateur (L'Ecole), au destinataire (l'étudiant potentiel), à l'objet (la formation). Ces caractéristiques étant dans ce contexte attachées à des représentations et à des valeurs.
} 
Ces formations peuvent être coordonnées par un enseignant permanent mais les intervenants sont surtout des professionnels d'entreprises ou des enseignants vacataires en droit, économie ou gestion.

La catégorie (3) désignée sous le terme de «Adaptabilité et citoyenneté » regroupe des programmes relevant d'une organisation proche de la catégorie précédente mais comportant un élargissement des enseignements, par une ouverture culturelle sur le monde contemporain et des disciplines telles que les lettres, les arts et parfois les SHS. Les intervenants peuvent être des enseignants permanents de l'École ou des vacataires. Les enseignements sont proposés sous forme d'options et portent sur des domaines variés. Les sciences dites de l'entreprise et le management conservent la meilleure place.

La catégorie (4) intitulée «Visée humanitaire » est voisine de la catégorie précédente par les enseignements proposés, mais elle met davantage l'accent sur des valeurs humaines de référence, le développement personnel et comportemental des étudiants. Il y est question des enjeux du monde contemporain, d'éthique, d'exemplarité et de dimension humaine des conduites professionnelles en entreprise, sans qu'il soit fait explicitement mention des SHS. Les intervenants sont souvent des enseignants permanents de l'École et l'accent est mis sur l'accompagnement des étudiants dans les choix qui orientent leur projet personnel et professionnel, ainsi que sur les activités associatives au sein des Écoles ou la gestion de projets humanitaires. Dans cette catégorie le curriculum « caché » revêt une dimension importante.

La catégorie (5) que nous avons désignée par les termes «Pluridisciplines et Humanités » accorde une place aux enseignements en SHS, mais aussi en lettres et arts. Il s'agit d'expliciter les enjeux liés à l'autonomie de pensée pour développer un esprit critique constructif sur les sciences et les techniques. Les savoirs dispensés sur les entreprises, le management, la gestion relèvent des SHS qui peuvent faire l'objet de travaux de recherche au sein même des Écoles. Les intervenants sont des enseignantschercheurs universitaires rattachés à des laboratoires de recherche dans des équipes associées. Les enseignements portent sur l'économie, l'histoire et la philosophie des sciences, la géopolitique, la sociologie, la psychologie, les sciences de gestion et les sciences politiques. Ils sont découplés des enseignements scientifiques et techniques.

La catégorie (6) appelée «Intégration en interdiscipline » dépasse l'objectif habituel d'acquisition des compétences spécifiques à la pratique du métier, apportées par les SHS ou les Humanités. Les sciences et les technologies sont analysées en tant que phénomènes complexes qui permettent de prendre en compte et de comprendre les systèmes d'interaction entre dimensions économiques, sociales, politiques, scientifiques, symboliques. Par la visée intégrative des connaissances qu'elle engage, cette catégorie renvoie à des formations qui tendent à construire une approche interdisciplinaire, entre SHS et sciences et techniques de spécialité, de ce qui est généralement qualifié de « cœur de métier » dans les pratiques professionnelles des ingénieurs. Les intervenants sont des enseignants-chercheurs universitaires rattachés à des laboratoires de recherche dans des équipes associées. Les enseignements disciplinaires (économie, ergonomie, sociologie par exemple) sont aussi intégrés dans des projets d'ingénieurs placés sous la double responsabilité des enseignants-chercheurs de SHS et de sciences et techniques de spécialité des ingénieurs.

Cette analyse empirique sur la place qu'occupent les SHS dans les curricula des formations d'ingénieurs en France tels qu'ils apparaissent sur les sites internet met en valeur leur position problématique. Dans la majorité des cas, les SHS n'apparaissent pas en tant que telles. Si la référence à l'entreprise domine dans les affichages des cursus, celle-ci ne semble que peu mise en perspective dans les différentes disciplines dont elle est l'objet et qui relèvent de la micro-économie, des sciences de gestion, de la sociologie, de la psychosociologie, de l'ergonomie ou encore des sciences du travail. A quelques exceptions près, la référence explicite aux SHS reste minoritaire. Lorsque c'est le cas, elles occupent des fonctions de sensibilisation, d'ouverture, de complément de formation sous forme de culture générale et/ou de formation morale. Avec de faibles volumes d'enseignement, elles sont 
généralement séparées des enseignements consacrés à l'acquisition des savoirs disciplinaires relatifs aux spécialités techniques. Les SHS ne sont explicitement et systématiquement mobilisées que dans deux catégories sur six: en tant que secteur de connaissance propre à enrichir une formation intellectuelle au même titre que les lettres et les arts (cat. 5); dans l'autre (cat. 6), regroupant très peu de cas, les SHS sont intégrées dans le cursus de formation sur des thématiques interdisciplinaires.

Bien que très marginale, la mise en évidence de cette dernière catégorie revêt un intérêt particulier au regard des travaux de recherche du projet européen HELENA (ibid.) puisque cette enquête, conduite dans différents pays européens et au Canada, montre l'influence positive des modules et des programmes interdisciplinaires dans les formations d'ingénieurs sur la motivation et la réussite des élèves-ingénieurs, en particulier des femmes. Le croisement des résultats de ces deux enquêtes ouvrent ainsi nombre de nouvelles interrogations, en particulier sur les pistes explicatives concernant la désaffection des formations par les femmes.

\section{La notion de " culture technique » en tant que facteur de différenciation}

Comment comprendre ces proximités, traduites dans cette enquête en regroupements par catégories, entre des Écoles si différentes ? Comment comprendre par ailleurs que les SHS soient si peu mobilisées en tant que telles dans la formation des ingénieurs? Comment comprendre enfin ce lien mis en valeur par l'enquête HELENA (ibid.) entre interdisciplinarité et genre?

\section{Une notion synthétique}

La sociologie du curriculum (Forquin, 2008) a montré que les choix réalisés dans l'organisation des cursus de formation sont socialement construits, en partie implicites, parfois contingents, relevant ainsi de ce que l'on pourrait qualifier de «culture » commune à une communauté académique et professionnelle, notion comprise dans son acception anthropologique en tant qu'ensemble partagé de savoirs, de codes, de valeurs, de représentations. Les Écoles d'ingénieurs étant fortement attachées à des domaines disciplinaires particuliers dont les aspects culturels et épistémologiques sont inséparables et entremêlés (Becher, 1994), il apparaît pertinent de se référer à la notion de " culture technique » (Godin et al., 1998). Ce simple syntagme permet en effet de prendre en compte de manière conjointe tout un ensemble d'éléments abordés habituellement de manière séparée : a) les savoirs, connaissances, compétences d'un domaine technique particulier ; b) mais aussi les savoir-faire, conduites et comportements, formes d'organisation et de fonctionnement, coutumes, habitus et habitudes qui sont attachés à l'exercice des activités humaines autour de ce domaine technique ; c) mais encore les symboles et croyances, systèmes de valeurs et représentations du monde, caractéristiques en des lieux et des temps donnés, les manières de penser et d'agir, les styles de vie d'une communauté par rapport à une autre. On peut ainsi remarquer des différenciations notamment à partir des appellations ${ }^{12}$ des enseignements de SHS ou assimilés dans le rapport aux savoirs ${ }^{13}$ produits par les SHS, dans la relation

\footnotetext{
12 Par exemple « connaissance de l'entreprise », « culture générale de l'ingénieur », «formation humaine », « formation humaine et sociale », « humanités », « technologie et sciences de l'homme », etc.

13 Variation par exemple de l'affichage plus ou moins valorisant des enseignements de SHS ou assimilés, le nom des Départements, l'existence même de recherches en SHS. On peut remarquer que les Départements de SHS et les recherches en SHS sont surreprésentés dans les Écoles des ministères techniques, les Écoles généralistes et celles des sciences de la vie et de la terre par rapport à d'autres tutelles et domaines de spécialité.
} 
avec des dimensions confessionnelles ${ }^{14}$ ou encore dans les rapports de genre ${ }^{15}$, selon les tutelles, le domaine de spécialité et les secteurs professionnels associés, la période historique d'ouverture de l'École ; ces critères étant parfois liés ${ }^{16}$.

Dans cette perspective, les programmes d'étude des Écoles d'ingénieurs, peuvent être considérés comme les vecteurs d'une transmission de cette culture technique partagée dans un milieu académique, scientifique et technique, mais aussi professionnel et industriel. Face à la diversité des possibles en termes de formation à des savoirs, des savoir-faire et des compétences, les Écoles se caractérisent donc par des positions épistémiques différentes qui se répercutent notamment sur la connaissance et la reconnaissance des SHS dans leur contribution possible à la formation des ingénieurs ${ }^{17}$. Sur ce point, la thèse ${ }^{18}$ d'A. Dufour ( 1998) est illustrative par sa mise en évidence, grâce à une étude socio-historique, des interrelations entre deux positions épistémiques différentes qui, en faisant système avec un ensemble de facteurs exogènes (représentations sociales et professionnelles, pressions des tutelles et des publics, critères de rentabilité économiques, etc.), ont débouché, dans deux périodes historiques différentes, sur deux projets très distincts d'organisation des formations relevant de deux cultures techniques opposées. Comprise dans sa double dimension structurelle (éléments stables dans la durée) et dynamique (soumise au changement, à la variabilité, à la diversité) (Desjeux, 2004), l'approche de la culture technique est plus aisée à conduire au macro-niveau des temporalités longues et des regroupements en grandes catégories.

\section{Une illustration de la notion}

Dans le domaine des sciences de la vie et de la terre, l'exemple des Écoles placées sous la tutelle du Ministère de l'agriculture est illustratif de la notion de culture technique.

On peut se référer à titre d'exemple aux Écoles d'ingénieurs agronomes, pour lesquelles le ministère de tutelle joue un rôle incontestable (Boulet, 1991, p. 19), précurseur dans plusieurs domaines: un enseignement mixte depuis 1917, alors que la généralisation de la mixité dans les Écoles d'ingénieurs en France ne date que de la loi Haby de 1974 ; la création en 1966, dans la mouvance de l'éducation populaire, d'un corps d'enseignants pour « l'éducation socio-culturelle » visant moins l'acquisition de connaissances que l'épanouissement des personnes en formation ; une politique d'appui aux mouvements associatifs au plan national et au plan local ; une rénovation de l'enseignement, en 1984, fruit d'un mouvement exceptionnel de réflexion et de débat. Plusieurs points spécifiques de la loi

\footnotetext{
14 Il semble que ce soient les héritages de la « doctrine sociale de l'Église » qui conduisent les Écoles catholiques à se référer à la notion de «formation humaine » (Didier, 2007). Dans la période 1830-1960, la hiérarchie de l’Église a été convoquée pour bénir les fruits de l'innovation technique : hauts-fourneaux, locomotives, gares, ponts suspendus, bateaux à vapeur, automobiles et aéroplanes (Lagrée, 1999). La bénédiction d'un monde industriel appelé à libérer l'homme moderne dans ses efforts pour imiter le Créateur s'accompagne d'une extension de la mission évangélisatrice. A la suite de Frédéric Le Play, l'Union sociale des ingénieurs catholiques (USIC) a contribué à promouvoir le rôle social de l'ingénieur au tout début du XXème siècle. C'est ainsi que s'instituent en France une approche mystique de la technique qui occulte le questionnement d'un autre rapport possible de l'homme à la technique.

15 S'il n'y a que $10 \%$ de femmes directrices d'Écoles d'ingénieurs, elles sont en revanche surreprésentées dans les Écoles ouvertes ces deux dernières décennies et dans les Écoles des domaines de spécialité liés à la chimie, aux sciences du vivant ou à l'administration. Cette observation est congruente avec d'autres travaux qui mettent en valeur dans certaines Ecoles et groupes professionnels associés « les traits symboliques et les pratiques de la domination masculine» (Marry, 2004, p. 67).

16 Par exemple, ce sont les Écoles du ministère de la Défense et les Écoles privées qui utilisent essentiellement l'appellation de « formation humaine, » en lien avec des appellations traditionnelles de «formation humaine et militaire » ou « formation humaine et religieuse ». Appellation qui tend à disparaître dans les Écoles les plus récentes qui préfèrent les termes de communication et de management.

${ }^{17}$ Les catégories proposées en sont des exemples, ces positions allant de quasiment pas de SHS du tout à une intégration des SHS en interdiscipline (les cas les plus fréquents étant ceux des catégories intermédiaires, «culture métier » et « adaptabilité et citoyenneté »).

18 Cette thèse est présentée dans un article soumis à la revue TransFormations : Roby C., Albero B., Formation des ingénieurs et SHS : les apports de deux thèses pionnières en sciences de l'éducation.
} 
d'orientation de l'enseignement agricole peuvent par ailleurs être notés, comme la participation des établissements privés au service public d'éducation et de formation ou encore la mission de recherche fondamentale et appliquée pour les Écoles d'ingénieurs privées sous contrat avec le ministère de l'agriculture. La première mission de l'enseignement agricole supérieur public est de « dispenser des formations scientifiques, techniques, économiques et sociales » dans les domaines des productions agricoles et de leur transformation et commercialisation, entre autres. Une mission qui témoigne du moindre écart entre savoirs académiques et expérience sociale, caractéristique des formations techniques du domaine (Boulet, 1991, p. 48-136). Ceci provient en partie du fait que l'agronomie qui est centrale dans ces formations d'ingénieurs, présente un caractère scientifique systémique qui exige une intégration interdisciplinaire, liée à sa finalité professionnelle et articulant des sciences et techniques de spécialité à des SHS (Prévost, Martinand, 2012). Des SHS dont la présence dans ces Écoles, économie et sociologie, est attestée dès 1948 (Cépède, Madec, 1966), et dont la mobilisation s'avère incontournable dans les recherches en agronomie et en sciences de l'environnement (Hubert, 2005, 2010 ; Desfontaines, Hubert, 2004 ; Létourneau, 2010). Une recherche qui «s'est toujours voulue finalisée [...] en prise avec les pratiques des agriculteurs » et dont les modes de polarisation couplés aux ordres socio-économiques de production expliqueraient les transformations récentes d'un secteur qui ne peut se comparer au monde industriel (Aggeri, Hatchuel, 2003).

Ces quelques éléments illustrent ce que peut signifier la notion de culture technique des Écoles d'ingénieurs, liée à un domaine particulier d'activité, à son histoire et à sa structuration en ordre socioéconomique de production, ainsi qu'au mode de polarisation de la recherche dans le domaine.

\section{Eléments de conclusion}

Les résultats obtenus, tant sur la possibilité de construction d'une catégorisation concernant la place et la fonction des SHS que sur la pertinence de la notion de culture technique pour caractériser les Écoles d'ingénieurs, sont encourageants pour justifier la reprise de la réflexion dans un domaine où la difficulté persistante à penser l'articulation entre formation scientifique et technique et formation aux disciplines des SHS présente un double intérêt épistémologique et social, axiologique et pragmatique.

Certes des questions subsistent. Par exemple, l'attention portée à la discipline principale de formation peut être questionnée, en regard notamment de la tension entre des enseignements de SHS à visée utilitaire, développementale ou dans des perspectives plus émancipatrices. Les approches présentées sont encore loin d'offrir une parfaite connaissance des différentes situations des SHS présentes dans les Écoles d'ingénieurs. On peut estimer le pourcentage des SHS dans les curricula, d'environ $10 \%$ à $40 \%$ selon les catégories et estimer ces pourcentages du même ordre pour les crédits ECTS. Mais au-delà de ces chiffres, le plus fondamental est certainement la reconnaissance accordée aux SHS dans les formations et leurs liens avec les disciplines des sciences et techniques de spécialité des Écoles d'ingénieurs. Pour cela, seules des enquêtes de terrain à l'échelle des Écoles peuvent permettre d'obtenir une connaissance plus précise des réalités de ces formations. On sait qu'elles sont dépendantes des contraintes locales auxquelles elles sont soumises, Michel Cottes (2013) l'a montré par une comparaison critique entre deux expériences très différentes de mise en place d'enseignements en SHS. On sait aussi que, malgré les raisons de diverse nature qui justifient l'intérêt d'un rapprochement entre sciences de spécialité et SHS dans la formation des ingénieurs, le constat d'une relation problématique demeure face à la rareté des Écoles qui investissent dans la formation interdisciplinaire et le renouvellement des pratiques de formation concernant ces enjeux. Pourtant, la prise au sérieux des travaux des SHS qui développent des connaissances pertinentes pour le champ de l'ingénierie, dans une période de complexification, d'accélération et d'internationalisation des problématiques techniques concernant le vivant, qu'il soit naturel et/ou social, pourrait offrir des opportunités et permettre aux Écoles de nombreuses initiatives en la matière. A titre d'exemple, dans la sixième version du document «Références et Orientations » publié en 2009 par la CTI, les objectifs de 
la formation sont présentés en trois points : 1) Acquisition et maitrise des connaissances scientifiques et techniques de base (générales, spécialité, ingénieur) ; 2) Adaptation aux champs professionnels nationaux et internationaux (entreprise, innovation et recherche) ; 3) Développement de la dimension humaine et sociétale (personnelle, sociale et environnementale). Ces objectifs renvoient à des qualités pouvant entrer en tension entre elles, par exemple dans la prise en compte des enjeux de compétitivité et de productivité et la mise en œuvre des principes du développement durable. Ces tensions seraient-elles moindres si la maîtrise des outils et méthodes de l'ingénieur passait par le développement de capacités à poser les problèmes dans toutes leurs dimensions, en tenant compte de leur contexte, avant de chercher à les résoudre techniquement ? Ou bien encore si la maitrise des connaissances scientifiques et techniques comportait une dimension épistémologique ? Ce seraient là des capacités que les SHS pourraient aider à acquérir. C'est pourquoi une plus grande lisibilité des offres et des modalités de formation en SHS des élèves-ingénieurs permettrait aux Écoles un meilleur positionnement, utile à leurs partenaires et aux futurs candidats.

Si l'on s'accorde à penser que l'enjeu principal des formations scientifiques et techniques ne se limite pas à l'acquisition stricte de savoirs et de savoir-faire réduits à ces champs, mais relève également du développement de dispositions à la responsabilité, à l'action stratégique et à la décision, dans la prise en compte réflexive des réalités de contexte et des caractéristiques de l'activité humaine, il apparaît alors pertinent de chercher à savoir quelle est la place accordée au développement de telles compétences dans les cursus. L'enquête empirique montre que les SHS peuvent assurer une fonction de temporisation du rythme de l'innovation, de prise de recul face à l'actualité, de problématisation face à l'accélération des changements techniques et économiques centraux dans la formation des ingénieurs. Celle-ci, à ces conditions, peut conduire à mieux comprendre le développement technique non comme simple moyen de résolution d'un problème, mais comme vecteur d'un projet social et politique dont les finalités, dans les pays à visées démocratiques, sont toujours à débattre.

\section{Références}

Albero B. (2010) Penser le rapport entre formation et objets techniques : repères conceptuels et épistémologiques. Dans G. Leclercq et R. Varga, Dispositifs de formation et environnements numériques : enjeux pédagogiques et contraintes informatiques. Paris: Hermès/Lavoisier, coll. IC2, 37-69.

Albero B. (2004) Travaux, interrogations et pistes de réflexion dans un champ de recherche éclaté. Note de synthèse. Savoirs, Technologies et formation, ${ }^{\circ} 5$, novembre, 11-69.

Aggeri F., Hatchuel A. (2003) Ordres socio-économiques et polarisation de la recherche dans l'agriculture : pour une critique des rapports science/société. Sociologie du Travail, 45(1), 113-133. doi:10.1016/S0038-0296(02)01308-0.

AERES (2010) Formation universitaire au métier d'ingénieur. Octobre. http://www. aeres-evaluation.fr/Actualites/Actualites-de-l-agence/ETUDE-Formation-universitaire-au-metier-d-ingenieur, (consulté le 4/05/2011).

Becher T. (1994) The Significance of Disciplinary Differences. Studies in Higher Education, 19(2), 151-161.

Bordier R., Kirchner A., Nussbaumer J. (2011) Adapter la formation de nos ingénieurs à la mondialisation. Institut Montaigne http://www.institutmontaigne.org/medias/ documents/etude_formation_ingenieurs-mondialisation.pdf, (consulté le 04/05/2012).

Boulet M. (1991) De l'enseignement agricole au savoir vert. Paris : Harmattan.

Cépède M., Madec A. (1966) Contribution de la sociologie a l'économie rurale. Économie rurale, 70(1), 3-14. doi:10.3406/ecoru.1966.1952.

Deffontaines J.-P., Hubert B. (2004) Dossier Interdisciplinarité Un regard sur l'interdisciplinarité à l'Inra. Natures Sciences Sociétés, 12(2), 186-190. doi:10.1051/nss:2004025. 
Cottes M. (2013, à paraître) Les sciences humaines et sociales entre utilité pratique et formation culturelle : l'exemple des Écoles d'ingénieurs. Actes du Colloque Les sciences humaines dans les parcours scientifiques et techniques professionnalisants : quelles finalités et quelles modalités pratiques? Université de Créteil, 7-8 février.

Desjeux D. (2004) Les sciences sociales (1re éd.) Paris : Presses universitaires de France.

Didier C. (2008) Penser l'éthique des ingénieurs. Paris : Presses universitaires de France.

Dufour A. (1998) Les enjeux de l'enseignement de la sociologie dans une École d'ingénieur. Analyse du curriculum de l'Institut supérieur d'agriculture Rhône-Alpes de 1968 à 1994. Thèse en sciences de l'éducation, université Lumières, Lyon 2.

Forquin J.C. (2008) Sociologie du curriculum. Rennes : Presses universitaires de Rennes.

Godfroy A.S. (2013, à paraître) Décrire et comparer des formations d'ingénieurs interdisciplinaires : enjeux méthodologiques et épistémologiques. Actes du Colloque Les sciences humaines dans les parcours scientifiques et techniques professionnalisants : quelles finalités et quelles modalités pratiques? Université de Créteil, 7-8 février.

Godin B., Gingras Y., Bourneuf É. Québec (Province) Ministère de l'industrie, du commerce, Québec (Province) Ministère de la culture et des communications \& Conseil de la science et de la technologie (Québec) (1998) Les indicateurs de culture scientifique et technique. Sainte-Foy : Conseil de la science et de la technologie. http://www4.bnquebec.ca/pgq/2005/3077070.pdf, (consulté le 20/05/2013).

Greimas A.J. (1966) Éléments pour une théorie de l'interprétation du récit mythique. Communications, vol. $8, n^{\circ} 1$, pp. 28-59.

Greimas A.J. (1966) Sémantique structurale. Recherche de méthode. Paris : Larousse.

Hubert B. (2005) L'interdisciplinarité sciences sociales/sciences de la nature dans les recherches sur problème. In Entre connaissance et organisation : l'activité collective (Lorino et Teulier., p. 133-155) Paris : La Découverte. http://www.cairn.info.scdbases.uhb.fr/article. php?ID_ARTICLE=DEC_LORIN_2005_01_0133 (Consulté le 21/05/2013).

Hubert B. (2010) L'agronomie, science de l'agriculture ? Le Mouvement Social, 233, 143. doi:10.3917/lms.233.0143.

ISAE Executive Club (2011) Réinventer le métier d'ingénieur pour en valoriser le rôle dans la société. Livre blanc. http://www.isae.fr/modules/ressources/download/default/Actualites/ doc/livre\%20blanc\%20ingenieur.pdf, (consulté le 04/05/2011).

Lagrée M. (1999) La bénédiction de Prométhée : religion et technologie, XIXe-XXe siécle. Paris : Fayard.

Lamard P., Lequin Y.-C. (2006) La technologie entre à l'université : Compiègne, Sevenans, BelfortMontbéliard. Sevenans : Université de technologie de Belfort-Montbéliard.

Lemaîitre D. (2003) La formation humaine des ingénieurs. Paris : Presses universitaires de France.

Lemaître D. (2001) Formation humaine dans les Écoles d'ingénieurs : étude des conceptions contemporaines. Thèse en sciences l'éducation, université de Nantes.

Létourneau A. (2010) La transdisciplinarité considérée en général et en sciences de l'environnement. VertigO - la revue électronique en sciences de l'environnement, Vol. 8, ${ }^{\circ}{ }^{\circ}$. doi : 10.4000/vertigo. 5253.

Le Play F. (2008) Naissance de l'ingénieur social : les ingénieurs des Mines et la science sociale au XIX ${ }^{\mathrm{e}}$ siécle. Paris : Mines-ParisTech.

Linard M. (1994) Vers un sujet de la connaissance dans les modélisations de l'apprentissage. Intellectica, vol. $2, \mathrm{n}^{\circ} 19,117-165$.

Linard M. (1989, 1996, nouv. éd. réactualisée) Des machines et des hommes. Apprendre avec les nouvelles technologies. Paris : L'Harmattan, coll. Savoir et formation.

Marry C. (2004) Les femmes ingénieurs : une révolution respectueuse. Paris : Belin. 
Les sciences humaines dans les parcours scientifiques et techniques professionnalisants

Prévost P., Martinand J.L. (2012) L'agronomie, une discipline d'enseignement technologique à enjeux didactiques. Présenté à XVIIème Congrès Mondial AMSE AMCE WAER Recherches en éducation et en formation : enjeux et défis aujourd'hui, Reims. http://www.lirdef.univmontp2.fr/node/313, (consulté le 20/05/2013).

Roby C. (2011) Vers une cartographie des places des sciences humaine et sociales dans les Écoles d'ingénieurs en France. Doctoriales internationales de l'éducation et de la formation, CREN, Nantes, France, 24-25 novembre 2011. [http: //www . cren . univ-nantes.fr/5464 . . ] ]

Rollet L. (2009) Peut-on faire l'histoire des pôles scientifiques ? Histoire de l'éducation, (122), 93-113.

Sainseaulieu Y., Jammet T. (2011) Les ingénieurs et leurs compétences non techniques. Lausanne : École Polytechnique fédérale (EPFL).

Sonntag M., Lemaître D., Fraysse B., Becerril R., Oget D. (2008) Les questions de formation dans les Écoles d'ingénieurs Un débat reconnu. Une place pour la recherche ? Recherches \& Educations, (1), 121-144.

Strauss A. (1992) La trame de la négociation : sociologie qualitative et interactionnisme. Paris : L'Harmattan.

Strauss A. (2004) Les fondements de la recherche qualitative ? : techniques et procedures de developpement de la theorie enracinee. Fribourg : Academic Press Fribourg.

Visier L. (2011) Vingt ans d'enseignement des sciences humaines et sociales dans les études médicales en France. Bioethica Forum, 4(4), 143-148. 